\title{
Adherencia médica a las indicaciones de procedimientos invasores en la unidad de pacientes críticos de un hospital universitario
}

\author{
L. Ricardo Gálvez, M. Angélica Berasain, Cecilia Luengo, Marcela Cifuentes, \\ Eduardo Tobar, M. Irene Jemenao y Francisco Silva
}

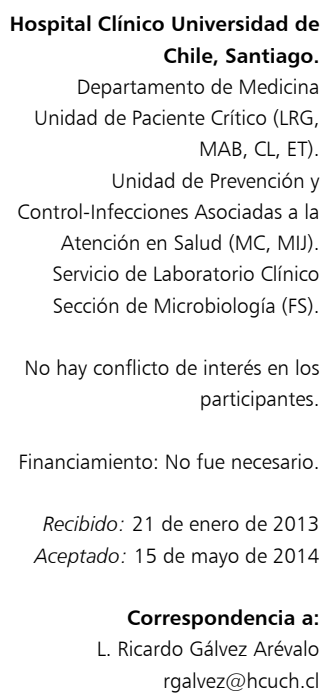

rgalvez@hcuch.cl

\author{
Physician's adherence to medical indications for invasive procedures in the critical \\ care unit of a university hospital
}

Introduction: Physicians' adherence to pre-established criteria for the indication and/or maintenance of invasive devices is a weak point in infection control programs. Fulfillment of the recommendations for preventing infections associated with invasive devices is essential to reduce their risk. Objective: To assess the adherence of physicians to the standardized criteria for indication of central venous catheter (CVC) and permanent urinary catheter (PUC) and to the application of supervision guidelines. Methods: During a period of 7 months, residents of the Critical Patient Unit monitored the adherence to criteria for indication of CVC and PUC recorded in patients' medical records. This information was compared with current regulations to assess compliance. Results: Between April and September 2009, 2078 supervision guidelines were applied. Invasive devices were identified in $47.7 \% .10 .4 \%$ of CVCs and $19.2 \%$ of PUCs did not meet criteria for installation and / or maintenance at the time of monitoring. Conclusions: Adherence of our medical staff to criteria for installation and /or maintenance of CVC and CUP should be improved. Monitoring can be efficiently performed by residents and could reduce infections associated with invasive procedures.

Key words: Healthcare associated infections, supervision, invasive procedures.

Palabras clave: Infecciones asociadas a atención en salud, supervisión, procedimientos invasores.

\section{Introducción}

$\mathrm{D}$ esde 1982, la Vigilancia de Infecciones Intrahospitalarias en Chile, actualmente Infecciones Asociadas a la Atención en Salud (IAAS), ha tenido una constante evolución. Hasta 1985, la recolección de datos se basaba en la notificación espontánea del personal clínico, permitiendo detectar menos de $20 \%$ de IAAS (vigilancia pasiva). En 1986 se modificó la pesquisa, pasando a ser realizada por profesionales capacitados, revisando fichas clínicas de pacientes con factores de riesgo (vigilancia activa selectiva), lo cual aumentó la pesquisa sobre $45 \%$ a nivel nacional. Esto demostró que la capacidad de detectar IAAS está directamente relacionada al tiempo de vigilancia disponible ${ }^{1-3}$.

La Unidad de Prevención y Control de IAAS del Hospital Clínico Universidad de Chile, se planteó como objetivo lograr $90 \%$ de cumplimiento para las medidas establecidas en prevención y control. De este modo, dentro de las estrategias locales de nuestra Unidad de Paciente Crítico (UPC), se hizo necesario conocer nuestra situación basal.

Los objetivos de este estudio fueron:
- Evaluar la adherencia de los médicos residentes de UPC a los criterios de indicación de dos procedimientos invasores, como son la instalación de un acceso venoso central (CVC) y de un catéter urinario a permanencia (CUP), cuyo objetivo es prevenir la infección del torrente sanguíneo asociado a CVC $(\text { ITS-CVC })^{4}$, e infección urinaria asociada a catéter urinario a permanencia (ITU-CUP) 5 .

- Evaluar la adherencia de los médicos residentes de UPC a aplicar pautas de supervisión de los criterios mencionados, habitualmente aplicadas por la Unidad de Prevención y Control de IAAS.

\section{Metodología}

Trabajo prospectivo observacional, realizado entre abril y octubre de 2009 en la UPC del Hospital Clínico Universidad de Chile.

A los 18 médicos residentes de UPC ( 6 residentes por unidad: Unidad de Cuidados Intensivos (UCI), Unidades de Intermedio Quirúrgico (IQ) e Intermedio Médico (IM)), todos médicos internistas que realizan rotativa de turno 28 
h contínuo, se les solicitó aplicar las pautas de supervisión de criterios de indicación de instalación y/o mantención de CVC y CUP. Dichas pautas debían ser aplicadas durante el transcurso de sus $24 \mathrm{~h}$ de turno, en todos los turnos correspondientes a un mes calendario, con posterioridad a una capacitación personalizada en la aplicación de las mismas $^{6,7}$. Se completaron como mínimo cuatro turnos supervisados por cada residente al mes, incluyendo todas las unidades de UPC.

Se supervisó la adherencia a los criterios de indicación de dos procedimientos invasores: instalación y/o mantención de CVC y de CUP en paciente crítico, de acuerdo a las pautas de $\mathrm{IAAS}^{6,7}$ que corresponden a ajustes locales de recomendaciones internacionales ${ }^{3,4,8}$ (Tabla 1).

Durante el período en evaluación no hubo recambio del personal de UPC, así como tampoco se realizó capacitación del personal o cambios en los protocolos de manejo estándar de los pacientes, que pudieran haber interferido con los criterios de indicación de los procedimientos monitorizados.

Los resultados se expresan en porcentaje de presentación y tasas, según corresponda.

\section{Resulltados}

Del total de residentes de UPC (n: 18), sólo dos (11\%) no cumplieron con las metas de supervisión programadas en el período supervisado. Se aplicó un total 2.078 pautas de supervisión, abarcando las tres unidades de UPC. La distribución global de pautas en relación al número de pacientes y a días de exposición se presenta en la Tabla 2.

Cabe destacar que dentro del período evaluado no se evidenciaron cambios en las tasas de infecciones asociadas a CVC y/o CUP en los pacientes de UPC. Se evidenció, por otra parte, una tendencia a la disminución del promedio de días de invasión, que para CVC fue de 0,9 días y para CUP 0,3 días (Figura 1).

\section{Catéter venoso central}

Se aplicaron 1.067 pautas de supervisión de la adherencia a indicaciones médicas de $\mathrm{CVC}$, según la normativa vigente $^{6,7}$. Del total de pautas aplicadas, en 47,9\% (511 pautas) se encontró invasión con un acceso venoso central: $41,3 \%$ en IM, 34,8\% en IQ y 66,9\% en UCI (Tablas $2-3$, Figura 2). De las pautas que informaron presencia de un acceso venoso central, 10,4\% no cumplía con criterios de indicación médica de invasión: $12,8 \%$ en IM, 16,2\% en IQ y $6,1 \%$ en UCI (Figura 3 ).

La adherencia a la normativa de indicaciones médicas de CVC fue de 89,6\%. La administración de soluciones o fluidos irritantes para vías de bajo flujo $(41,5 \%)$ y la indicación de monitoreo hemodinámico $(29,9 \%)$, representaron la mayor parte de las indicaciones de invasión. La ausencia de accesos periféricos fue una indicación que no sobrepasó el 20\% de las pautas evaluadas (Tabla 3). Dentro de las pautas de supervisión que tenían invasión pese a no cumplir criterios de indicación médica, en 24/53 (45\%) tampoco se había indicado retiro del dispositivo, pese a no estar utilizándose al momento de la supervisión (Tabla 4). Del resto de pautas que no cumplía con las indicaciones de IAAS (29/53), un grupo presentó otros criterios de indicación médica, los cuales no están incluidos dentro de las recomendaciones IAAS vigentes (Tabla 4).

Tabla 1. Recomendaciones locales de indicación de procedimientos invasores, según norma vigente en el Hospital Clínico Universidad de Chile ${ }^{6,7}$, período 2008-2011

\begin{tabular}{|c|c|c|}
\hline \multirow{4}{*}{$\begin{array}{l}\text { A. Catéter veno- } \\
\text { so central }\end{array}$} & 1 & Ausencia de acceso venoso periférico \\
\hline & 2 & $\begin{array}{l}\text { Necesidad de monitoreo de presión venosa central (PVC) y/o saturación } \\
\text { venosa de oxígeno }\left(\mathrm{Sat}_{\mathrm{v}} \mathrm{O}_{2}\right)\end{array}$ \\
\hline & 3 & $\begin{array}{l}\text { Necesidad de administrar soluciones o fármacos que no se pueden infundir } \\
\text { en venas de bajo flujo: fármacos vasoactivos, nutrición parenteral, quimiote- } \\
\text { rapias, medicamentos irritantes }\end{array}$ \\
\hline & 4 & $\begin{array}{l}\text { Realización de procedimiento dialítico de apoyo en paciente con falla renal } \\
\text { aguda o crónica, que requiera reemplazo transitorio o definitivo de la fun- } \\
\text { ción renal }\end{array}$ \\
\hline \multirow{7}{*}{$\begin{array}{l}\text { B. Catéter urina- } \\
\text { rio permanente }\end{array}$} & 1 & Uropatía obstructiva \\
\hline & 2 & Vejiga neurogénica \\
\hline & 3 & Necesidad de efectuar irrigaciones vesicales \\
\hline & 4 & Post-operatorio de cirugía urológica \\
\hline & 5 & Pre y post-operatorio de cirugía pelviana \\
\hline & 6 & $\begin{array}{l}\text { Monitoreo de diuresis en pacientes con inestabilidad hemodinámica ( } 24 \text { h } \\
\text { con uso de fármacos vasoactivos o medición de parámetros de perfusión) }\end{array}$ \\
\hline & 7 & Pacientes bajo sedación profunda o bloqueo neuromuscular \\
\hline
\end{tabular}

Tabla 2. Distribución de pautas de supervisión en referencia al universo encuestado en UPC, período abril-octubre de 2009

\begin{tabular}{lcrrc} 
& UPC total & UCI & IM & IQ \\
Catéter venoso central & & & & \\
Días de exposición & 8.079 & 3.956 & 1.270 & 2.853 \\
Pautas realizadas & 1.067 & 371 & 322 & 374 \\
Pautas con invasión & 511 & 248 & 133 & 130 \\
Supervisión (\%) & 13,2 & 9,4 & 25,4 & 13,1 \\
Tasa de supervisión por 1.000 días de exposición & 132,1 & 93,8 & 253,5 & 131,1 \\
Tasa de invasión por 1.000 días de exposición & 63,3 & 62,7 & 104,7 & 45,6 \\
Catéter urinario permanente & & & & \\
Días de exposición & 7.685 & 3.243 & 1.324 & 3.118 \\
Pautas realizadas & 1.011 & 328 & 318 & 365 \\
Pautas con invasión & 480 & 204 & 137 & 139 \\
Supervisión (\%) & 13,2 & 10,1 & 24 & 11,7 \\
Tasa de supervisión por 1.000 días de exposición & 131,6 & 101,1 & 240,2 & 117,1 \\
Tasa de invasión por 1.000 días de exposición & 62,5 & 62,9 & 103,5 & 44,6 \\
\hline
\end{tabular}

Los días de exposición en las diferentes unidades de UPC fueron obtenidos de la vigilancia y supervisión continua que realiza la Unidad de Prevención y Control-IAAS del Hospital Clínico Universidad de Chile. UPC: Unidad de paciente crítico. UCl: Unidad de cuidados intensivos. IM: Intermedio médico. IQ: Intermedio quirúrgico. 

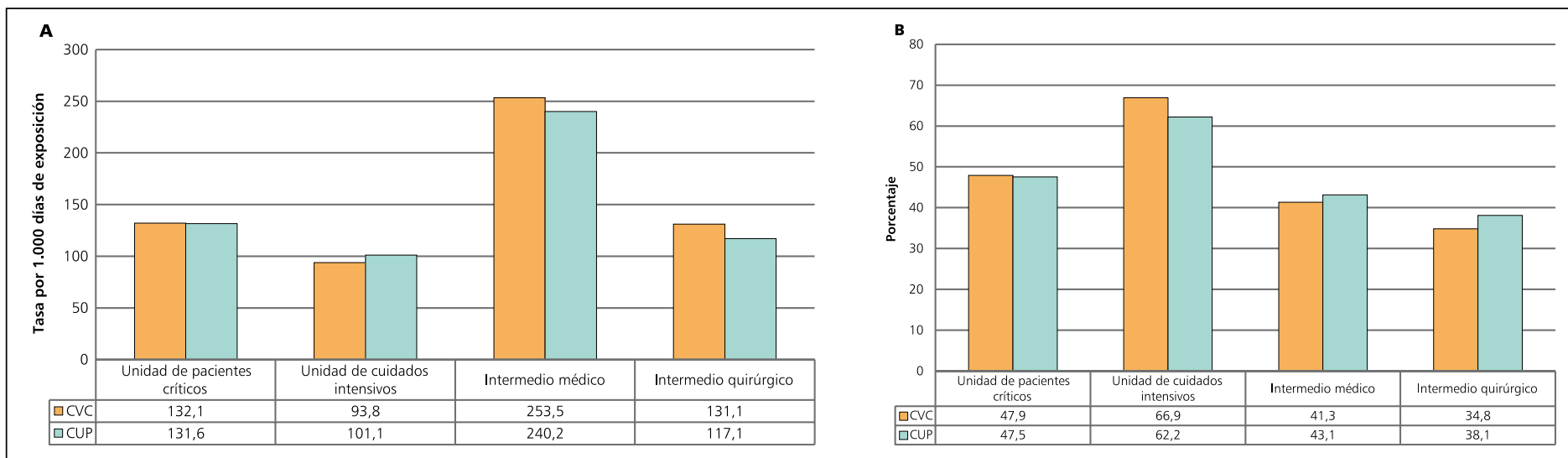

Figura 1. Distribución en tasas de supervisión (A) y porcentajes de invasión (B), de la indicación de catéter venoso central (CVC) y catéter urinario a permanencia (CUP) en las diferentes unidades de la Unidad de Pacientes Críticos (UPC), período Abril-Octubre 2009.

\section{Tabla 3. Distribución de pautas de supervisión y de indicación médica} de catéter venoso central, período abril-octubre de 2009

\begin{tabular}{|c|c|c|c|c|c|c|c|c|}
\hline & \multicolumn{2}{|c|}{ UPC } & \multicolumn{2}{|c|}{ UCI } & & \multicolumn{2}{|r|}{ o } \\
\hline Con invasión & 511 & 47,9 & 248 & 66,9 & 133 & 41,3 & 130 & 34,8 \\
\hline Criterio A1 & 85 & 16,6 & 18 & 7,3 & 46 & 34,6 & 21 & 16,2 \\
\hline Criterio A2 & 153 & 29,9 & 85 & 34,3 & 26 & 19,6 & 42 & 32,3 \\
\hline Criterio A3 & 212 & 41,5 & 125 & 50,4 & 44 & 33,1 & 43 & 33,1 \\
\hline Criterio A4 & 8 & 1,6 & 5 & 2 & 0 & 0 & J & 0,8 \\
\hline Sin indicación & 53 & 10,4 & 15 & 6,1 & 17 & 12,8 & 21 & 16,2 \\
\hline Sin invasión & 556 & 52,1 & 123 & 33,1 & 189 & 58,7 & 244 & 65,2 \\
\hline Total & 1.067 & 100 & 371 & 100 & 322 & 100 & 374 & 100 \\
\hline
\end{tabular}

Los valores (número y porcentaje) de cada criterio, así como los correspondientes al ítem "Sin indicación" están calculados en base al valor total de pautas con invasión. Los criterios de invasión corresponden a los mencionados en la Tabla 1. UPC: Unidad de paciente crítico. UCl: Unidad de cuidados intensivos. IM: Intermedio médico. IQ: Intermedio quirúrgico.

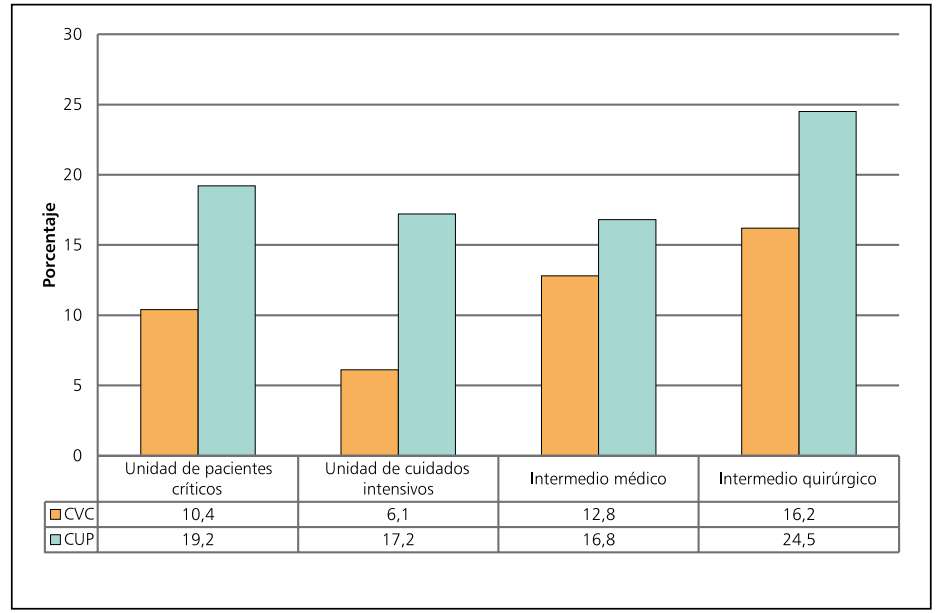

Figura 2. Distribución de porcentajes del no cumplimiento de indicación médica (criterios) para la instalación de catéter venoso central (CVC) y catéter urinario a permanencia (CUP), en período Abril-Octubre 2009.

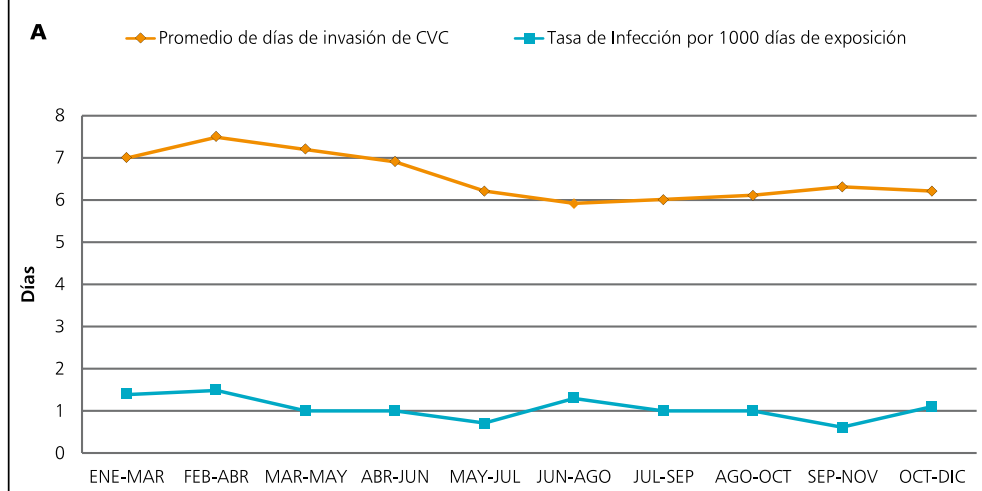

B $\rightarrow$ Promedio de días de invasión de CUP $\rightarrow$-Tasa de Infección por 1000 días de exposición

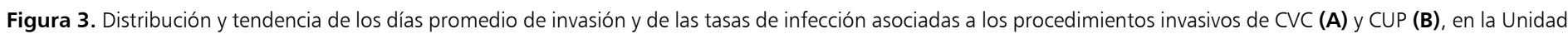

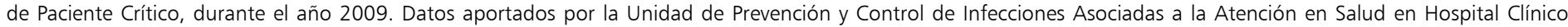
Universidad de Chile, presentados como trimestres móviles. 
Tabla 4. Distribución de las causas de indicación de catéter venoso central y catéter urinario a permanencia en el universo encuestado, no incluidas en las recomendaciones de utilización expuestas en Tabla 1

\section{Indicación}

Catéter venoso central

Post-operatorio de cirugía mayor

Terapia de reemplazo renal

Circulación extracorpórea (ECMO)

Sin uso, no explicita indicación de retiro

\begin{tabular}{rrrrr}
$\mathbf{n}$ & $\mathbf{( \% )}$ & $\begin{array}{l}\text { Catéter urinario a permanencia } \\
\text { Indicación }\end{array}$ & $\mathbf{n}$ & $\mathbf{( \% )}$ \\
11 & $(20)$ & Síndrome hépato-renal & 6 & $(6,9)$ \\
16 & $(30)$ & Monitoreo de Infusión continua de diuréticos & 12 & $(13,8)$ \\
2 & $(5)$ & Fase poliúrica de falla renal aguda & 38 & $(41,4)$ \\
24 & $(45)$ & Post-operatorio de cirugía mayor & 12 & $(13,8)$ \\
& $\quad$ Post-operatorio de cirugía traumatológica mayor & 12 & $(13,8)$ \\
& $\quad$ Otros * & 11 & $(10,5)$ \\
53 & $\quad$ Total & 92 & $(100)$ \\
\hline
\end{tabular}

\begin{tabular}{|c|c|c|c|c|c|c|c|c|}
\hline & \multicolumn{2}{|c|}{ UPC } & \multicolumn{2}{|c|}{ UCI } & \multicolumn{2}{|c|}{ IM } & \multicolumn{2}{|c|}{ IQ } \\
\hline & $\mathbf{n}$ & $\%$ & $\mathbf{n}$ & $\%$ & $\mathbf{n}$ & $\%$ & $\mathbf{n}$ & $\%$ \\
\hline Con invasión & 480 & 47,5 & 204 & 62,2 & 137 & 43,1 & 139 & 38,1 \\
\hline Criterio B1 & 17 & 3,5 & 1 & 0,5 & 9 & 6,6 & 7 & 5 \\
\hline Criterio B2 & 14 & 2,9 & 2 & 0,8 & 4 & 2,9 & 8 & 5,8 \\
\hline Criterio B3 & 13 & 2,7 & 0 & 0 & 8 & 5,8 & 5 & 3,6 \\
\hline Criterio B4 & 20 & 4,2 & 2 & 0,8 & 3 & 2,2 & 15 & 10,8 \\
\hline Criterio B5 & 5 & 1 & 0 & 0 & 0 & 0 & 5 & 3,6 \\
\hline Criterio B6 & 269 & 56 & 128 & 62,7 & 81 & 59,1 & 60 & 43,2 \\
\hline Criterio B7 & 50 & 10,4 & 36 & 17,6 & 9 & 6,6 & 5 & 3,6 \\
\hline Sin indicación & 92 & 19,2 & 35 & 17,2 & 23 & 16,8 & 34 & 24,5 \\
\hline Sin invasión & 531 & 52,5 & 124 & 37,8 & 181 & 56,9 & 226 & 61,9 \\
\hline Total & 1.011 & 100 & 328 & 100 & 318 & 100 & 365 & 100 \\
\hline
\end{tabular}

Un 52,1\% de las pautas no mostró invasión (556 pautas), 58,7 y $65,2 \%$ en las Unidades de IM e IQ, respectivamente, y $33,1 \%$ (123 pautas) se encontraron sin invasión en UCI al momento de la supervisión. No se demostró indicación de invasión en ellas (Tabla 3).

\section{Catéter urinario a permanencia}

Se aplicaron 1.011 pautas de supervisión de adherencia a indicaciones médicas de CUP, según las recomendaciones de IAAS ${ }^{6,7}$. Del total de pautas aplicadas, 47,5\% (480 pautas) presentaba invasión con CUP (Tabla 2, Figuras $1-2)$. El porcentaje de invasión en UCI fue $62,2 \%$, en IM $43,1 \%$ y en IQ $38,1 \%$ (Tabla 5). De las pautas con invasión por CUP, $19,2 \%$ no cumplía criterios de indicación médica de invasión al momento de la supervisión: $17,2 \%$ en UCI, $16,8 \%$ en IM y $24,5 \%$ en IQ (Tabla 5 , Figura 3). La adherencia a la normativa de indicaciones médicas de CUP fue de 80,8\%. Dentro de las pautas de supervisión que tenían invasión pese a no cumplir criterios de indicación médica de invasión, una de 92 pautas $(1,1 \%)$ tampoco tenía indicación de retiro, pese a no estar utilizándose al momento de la supervisión (Tabla 4). Del resto de pautas que no cumplían con las indicaciones de IAAS (91/92), un grupo presentó otros criterios de indicación médica, los cuales no están incluidos dentro de las recomendaciones IAAS vigentes. Dentro de estos, la causa más frecuente fue el monitoreo de la fase poliúrica de la falla renal aguda. Destacó también la monitorización 
post-operatoria de la cirugía mayor, tanto abdominal como traumatológica, y la monitorización del efecto de infusión continua de diuréticos (Tabla 4).

En 56,9\% del IM, 61,9\% del IQ y $37,8 \%$ en UCI, no se evidenciaba invasión al momento de la supervisión; ninguna de ellas tenía indicación de hacerlo (Tabla 5).

\section{Discusión}

La supervisión de procedimientos en medicina, con el fin de vigilar los riesgos asociados a IAAS, sólo cuenta con un formato protocolizado desde $1986^{1,2}$. Según las recomendaciones del Ministerio de Salud ${ }^{1}$, este protocolo conlleva la necesidad de un profesional de salud a su cargo en forma exclusiva, cuya función es la vigilancia epidemiológica de la instalación y mantención de los procedimientos invasores y las infecciones asociadas a ellos. La supervisión directa, sistemática y mantenida en el tiempo de la adherencia a las medidas de prevención y control de IAAS queda a cargo de las Jefaturas de Servicio. En Chile es habitual contar con buenos programas de supervisión en enfermería cuyos resultados han permitido evaluar tendencias en IAAS. Sin embargo, esto no se repite en el ámbito médico.

Este estudio permitió conocer la realidad de la UPC de un hospital universitario en cuanto a la adherencia médica a las normativas de prevención de IAAS; los resultados fueron enriquecedores. El traspasar la responsabilidad de dicha supervisión al personal médico a cargo representó un fuerte llamado de atención para el equipo de supervisores, quienes hasta esa fecha no evaluaban la justificación de la invasión dentro del algoritmo de manejo del paciente crítico. También definió un cambio importante en la visualización y evaluación diaria de los pacientes, quienes resultaron los más beneficiados.

De los resultados obtenidos, es importante destacar la existencia de 10,4 y 19,2\% de pacientes en UPC, que tenían invasión con CVC y CUP, respectivamente, a pesar de no tener indicación médica de procedimiento invasor al momento de la supervisión (Figura 3), según la normativa vigente.

Del 19,2\% de CUP sin indicación médica, sólo 1,1\% carecía de justificación real para permanecer con dicha invasión al momento de la supervisión. En el resto de los pacientes se pesquisaron otras indicaciones médicas de invasión no incluidas dentro de las recomendaciones vigentes a la fecha del estudio, pero que justificaban el uso de CUP (Tabla 4). De este modo, con posterioridad a una evaluación y discusión en conjunto con la Unidad de Prevención y Control de IAAS, estas indicaciones fueron incluidas en los criterios de invasión para CUP.

En referencia exclusiva a CVC, el riesgo potencial de infección asociado a su permanencia, hace inaceptable el
$10,4 \%$ de ausencia de adherencia a la indicación médica de invasión. Las unidades de intermedio mantienen un porcentaje de adherencia similar (Figuras 2-3). No obstante, de este $10,4 \%$, la presencia de otras indicaciones médicas de invasión, no incluidas en la normativa vigente (Tabla 4), hace que sólo 4,7\% no haya tenido justificación real de permanecer con CVC al momento de la supervisión. Aun así, sigue siendo una cifra preocupante que debe motivar intervenciones que la disminuyan con miras a reducir el riesgo de ITS-CVC.

Basándonos en nuestros resultados, una evaluación diaria y el retiro precoz de CUP y CVC, disminuiría teóricamente el riesgo de complicaciones asociadas, sobre todo de origen infeccioso, materia que no es objetivo de estudio en esta supervisión.

La experiencia de utilizar a nuestro personal médico residente como ente supervisor, nos permitió ser más críticos con las indicaciones, y al mismo tiempo, definir el retiro de dichas invasiones en forma más precoz, cuando, utilizando el criterio clínico, no existía ninguna indicación de mantenerlo. En este estudio logramos una adherencia médica a la supervisión de indicación de invasión de $89 \%$ (16/18). Si bien es un dato diagnóstico, consideramos que es un porcentaje alto teniendo en cuenta la falta de educación en relación a la importancia de la supervisión en el estamento médico.

Esta estrategia constituye una novedad dentro de la vigilancia epidemiológica de la adherencia a las indicaciones médicas de procedimientos invasores, habitualmente realizada por los Comités de IAAS. Por ser una experiencia innovadora, no logramos encontrar en literatura médica nacional o internacional, datos similares a los expresados en nuestro estudio.

Creemos que estas medidas, simples de instaurar, generan una ganancia y enriquecen el quehacer clínico hacia nuestros pacientes, con una vigilancia directa como tarea transversal del equipo de salud. La simple medida de derivar la responsabilidad de supervisión a los residentes médicos, permitió cambiar su visión clínica de los pacientes, generando al mismo tiempo en ellos, una actitud reflexiva y crítica en relación a los dispositivos invasores.

Basados en estos resultados, creemos que la realización de un programa de educación continua, enfocado directamente a la importancia de la supervisión de indicación y/o mantención de dispositivos invasores en pacientes críticos, nos permitiría aumentar a 100\% la adherencia de nuestros residentes a dicha supervisión. Esta intervención podría llevar a una disminución de la indicación y/o mantención de dispositivos invasores $\mathrm{y}$, eventualmente, a una disminución de las IAAS secundarias a ellos.

En referencia a las tasas de IAAS, si bien al evaluarlas en trimestres móviles existe una disminución en ellas, ésta aún no constituye una tendencia significativa (Figura 1), y su evaluación escapa a los objetivos del presente estudio. 
En conclusión, la supervisión directa y continua de la adherencia a las indicaciones médicas de procedimientos invasores como CVC y CUP, permite disminuir la permanencia innecesaria de estos dispositivos. Esto pudiera traducirse, en forma secundaria, en una disminución de complicaciones infecciosas, lo cual constituye el objetivo primario de la supervisión de IAAS. Esta experiencia nos permitió determinar que la supervisión directa por médicos residentes puede ser aún más efectiva y estricta que la supervisión por el personal de IAAS.

Agradecimientos. Al Equipo de Residentes de la Unidad de Paciente Crítico, por el apoyo en esta tarea de supervisión y al Equipo de Infecciones Asociadas a la Atención en Salud, por las directrices y recomendaciones al momento de definir nuestro trabajo.

\section{Resumen}

Introducción: La adherencia médica a los criterios de indicación y/o mantención de procedimientos invasores es un punto débil en los programas de control de infecciones.
Cumplir las recomendaciones relacionadas a prevención de infecciones asociadas a procedimientos invasores es fundamental para reducir el riesgo de infección. Objetivos: Evaluar la adherencia de médicos residentes a criterios estandarizados de indicaciones médicas de catéter venoso central (CVC) y catéter urinario permanente (CUP) y a aplicar pautas de supervisión para evaluar su cumplimiento. Método: Durante un período de siete meses, residentes de la Unidad de Paciente Crítico (UPC), monitorizaron la adherencia a los criterios de indicación de CVC y CUP registrados en las fichas clínicas de los pacientes. La información registrada se cotejó con la normativa vigente. Resultados: Entre abril y octubre de 2009 se aplicaron 2.078 pautas de supervisión, de las cuales $47,7 \%$ identificaron invasión al momento de aplicarlas. Un 10,4 y 19,2\% de los CVC y CUP, respectivamente, no cumplían con criterios de instalación y/o mantención al momento de la supervisión. Conclusiones: La adherencia de nuestros médicos de UPC a los criterios de instalación y/o mantención de CVC y CUP debe mejorarse. La supervisión puede ser realizada eficientemente por los mismos residentes y podría reducir las infecciones asociadas a procedimientos invasores.

\section{Referencias bibliográficas}

1.- Proyecto MINSAL/PNUD/OPS 1986-1990. www.minsal.cl; (último acceso 14 de octubre de 2012).

2.- Ajenjo M C. Infecciones Intrahospitalarias: Conceptos actuales de prevención y control. Revista Chilena de Urología 2006; 71 (2): 95-101.

3.- Yokoe D S, Mermel L A, Anderson D J, Arias K M, Burstin H, Calfee D P, et al. A compendium of strategies to prevent healthcare-associated infections in acute care hospitals. Infect Control Hosp Epidemiol
2008; 29 (suppl 1): S12-S21.

4.- Marschall J, Mermel L A, Classen D, Arias K M, Podgorny K, Anderson D J, et al. Strategies to prevent central line-associated bloodstream infections in acute care hospitals. Infect Control Hosp Epidemiol 2008; 29 (suppl 1); S22-S30.

5.- Lo E, Nicolle L, Classen D, Arias K M, Podgorny K, Anderson D J, et al. Strategies to prevent catheter-associated urinary tract infections in acute care hospitals. Infect Control Hosp Epidemiol 2008; 29 (suppl 1): S41-S50.

6.- Fica A, Ruiz G, Jemenao M I. Manual de
Control de Infecciones Intrahospitalarias, Productos Farmacéuticos y Normas Transfusionales. Hospital Clínico Universidad de Chile 2004-2007.

7.- Fica A, Ruiz G, Jemenao M I, Bilbao P. Control y Prevención de Infecciones Intrahospitalarias, Guías Transfusionales y Productos Farmacéuticos 2008-2011. Hospital Clínico Universidad de Chile.

8.- Smith P W, Bennett G, Bradley S, Drinka P, Lautenbach E, Marx J, et al. SHEA/APIC Guideline: Infection prevention and control in the long-term care facility. Infect Control Hosp Epidemiol 2008; 29 (9): 785-814. 